\title{
A fatal case of Emergomyces pasteurianus pulmonary infection in an immunocompromised patient with concomitant listeriosis and invasive aspergillosis
}

\section{Introduction}

Immunocompromised patients can become accidental hosts of dimorphic fungi by inhaling dust-borne conidia of fungal species belonging to the genus Emergomyces. Emergomyces is a recently proposed new genus of saprophytic dimorphic fungi related to the genus Emmonsia that are distributed worldwide and of which the exact environmental or animal source is still unknown. The genus Emergomyces contains two species: $E$. pasteurianus and $E$. africanus. These species have been found in human cases of disseminated infection in immunocompromised hosts, mainly in South Africa. Both new species lack adiaspores, classically associated with the genus Emmonsia. We report an E. pasteurianus pulmonary infection in an immunocompromised patient

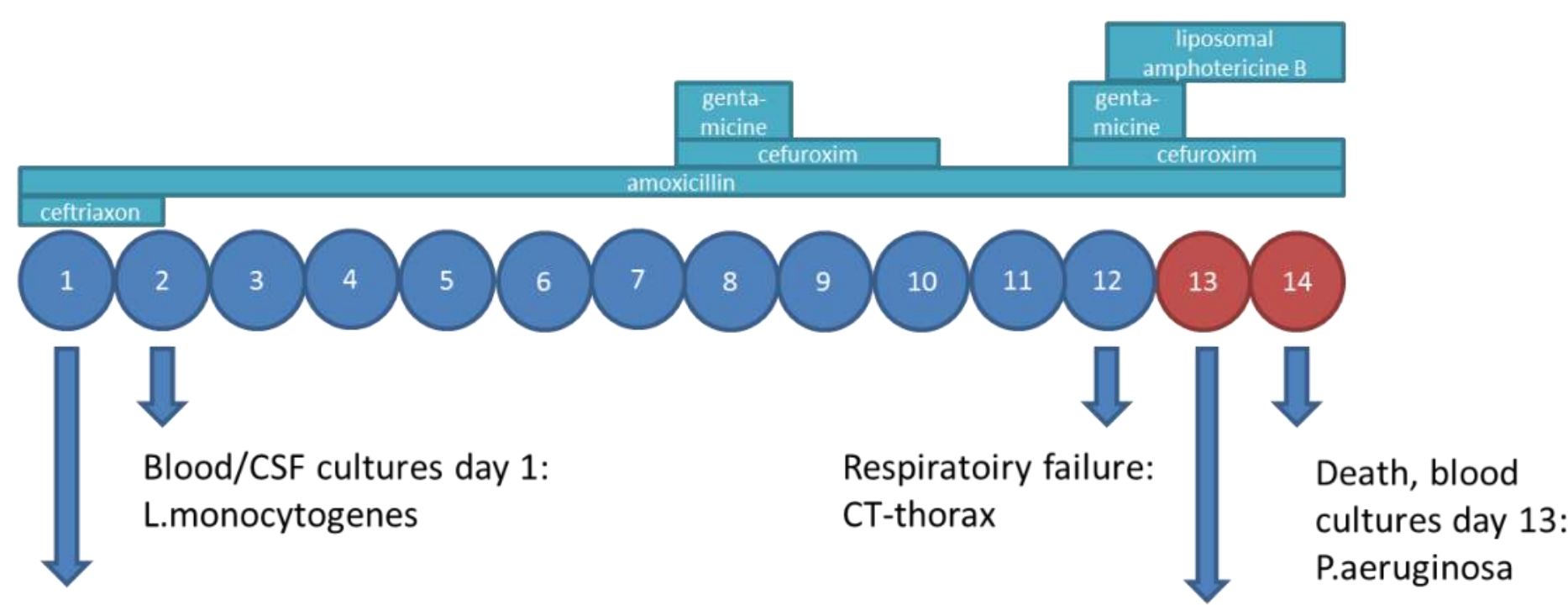

Admission: decreased level of consciousness, fever
Admission Intensive Care: respiratoiry failure, cardiac failure, sepsis $\rightarrow$ Broncho-Alveolar Lavage

\section{Clinical presentation}

Patient: history of B-CLL and chronic kidney failure.

Immunocompromised: chronic neutropenia due to his B-CLL and therapy (cyclophosphamide and prednisone since 11 months) Travelling: family visit to Iraq two months ago

Symptoms: tachypnea and persisting fever during treatment for Listeria meningitis with neurological improvement Imaging: CT-thorax on day 12: Bilateral extensive infiltrative abnormalities, suggestive for fungal infection, see figure 1 Treatment: empirical therapy with liposomal amphotericine B $3 \mathrm{mg} / \mathrm{kg}$ was started on day 12

Outcome: The patient died within $36 \mathrm{~h}$ after onset of tachypnea, because of respiratory insufficiency, cardiac failure and sepsis caused by Pseudomonas aeruginosa.

\section{Fungal diagnostics}

- Galactomannan assay (Platelia Aspergillus, Bio-Rad):

- Serum day 12 and BAL fluid day 13: strongly positive

- PCR A.fumigatus (ErasmusMC, Rotterdam, the Netherlands)

- BAL fluid day 13: positive

- Culture BAL fluid day 13

- No Aspergillus could be cultured

- After 3days growth of some small colonies suspected for a fungus. After three weeks of incubation, a dimorphic fungus was diagnosed without production of adiaspores at $28^{\circ} \mathrm{C}$.

In house ITS sequencing

- Dimorphic fungus: Emergomyces pasteurianus

ITS and LSU sequencing (CBS-KNAW Fungal Biodiversity Centre, Utrecht, The Netherlands)

- Dimorphic fungus: Emergomyces pasteurianus
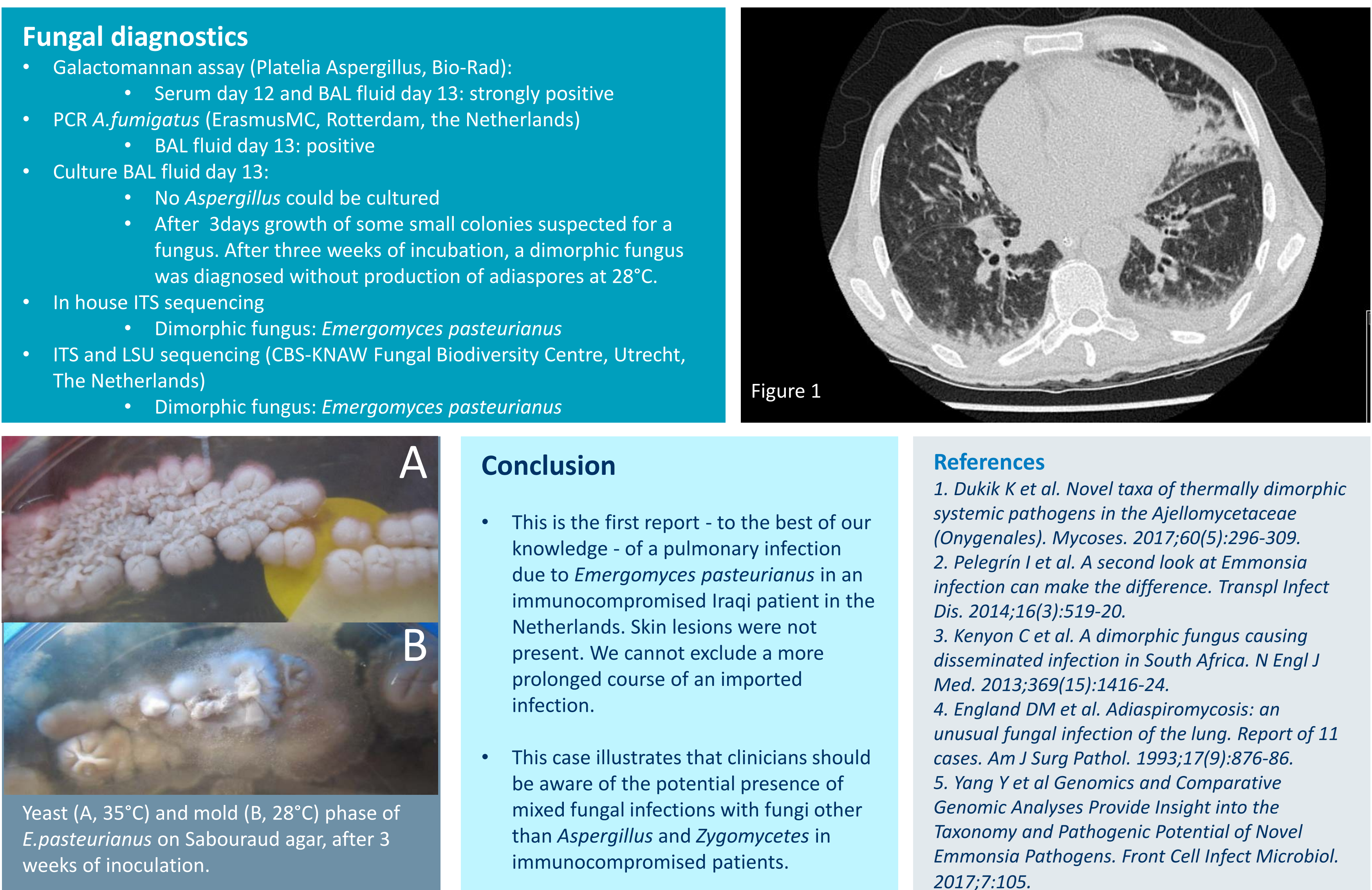

\section{Conclusion}

- This is the first report - to the best of our knowledge - of a pulmonary infection due to Emergomyces pasteurianus in an immunocompromised Iraqi patient in the Netherlands. Skin lesions were not present. We cannot exclude a more prolonged course of an imported infection.

- This case illustrates that clinicians should be aware of the potential presence of mixed fungal infections with fungi other than Aspergillus and Zygomycetes in immunocompromised patients.
References

1. Dukik K et al. Novel taxa of thermally dimorphic systemic pathogens in the Ajellomycetaceae (Onygenales). Mycoses. 2017;60(5):296-309. 2. Pelegrín l et al. A second look at Emmonsia infection can make the difference. Transpl Infect Dis. 2014;16(3):519-20.

3. Kenyon $\mathrm{C}$ et al. A dimorphic fungus causing disseminated infection in South Africa. N Engl J Med. 2013;369(15):1416-24.

4. England DM et al. Adiaspiromycosis: an unusual fungal infection of the lung. Report of 11 cases. Am J Surg Pathol. 1993;17(9):876-86. 5. Yang Y et al Genomics and Comparative Genomic Analyses Provide Insight into the Taxonomy and Pathogenic Potential of Novel Emmonsia Pathogens. Front Cell Infect Microbiol. 2017;7:105. 